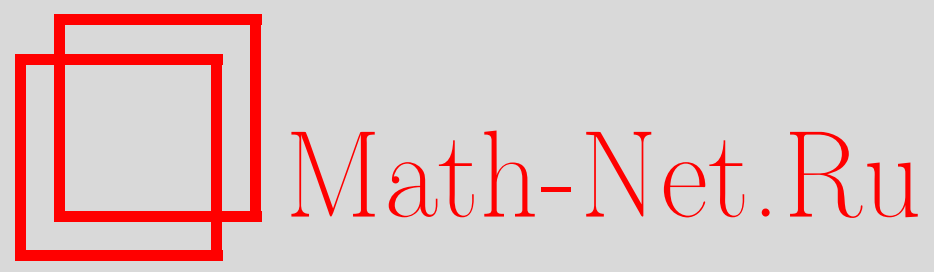

К. Г. Омельянов, А. А. Сапоженко, О числе множеств, свободных от сумм, в отрезке натуральных чисел, Дискрет. матем., 2002, том 14, выпуск 3, 3-7

DOI: https://doi.org/10.4213/dm248

Использование Общероссийского математического портала Math-Net.Ru подразумевает, что вы прочитали и согласны с пользовательским соглашением http://www.mathnet.ru/rus/agreement

Параметры загрузки:

IP : 54.224 .60 .19

26 апреля 2023 г., 16:33:05

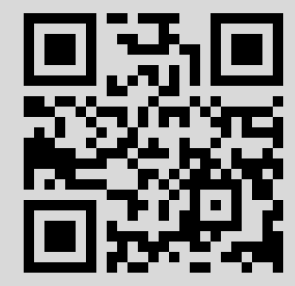


УдК 511.2

\title{
О числе множеств, свободных от сумм, в отрезке натуральных чисел
}

\author{
(c) 2002 г. К. Г. Омельянов, А. А. Сапоженко
}

Подмножество $A$ целых чисел называется свободным от сумм, если для любых $a, b \in A$ число $a+b$ не принадлежит множеству $A$. Для произвольного $\varepsilon>0$ обозначим $s_{\varepsilon}(n)$ число множеств, свободных от сумм, в отрезке $[(1 / 4+\varepsilon) n, n]$. В статье доказывается, что для всякого $\varepsilon>0$ существует постоянная $c=c(\varepsilon)$ такая, что $s_{\varepsilon}(n) \leqslant c 2^{n / 2}$.

Работа выполнена при поддержке Российского фонда фундаментальных исследований, проект 01-01-00266.

\section{1. Введение}

Подмножество $A$ целых чисел называется свободным от сумм, если для любых $a, b \in A$ число $a+b$ не принадлежит множеству $A$. П. Камерон в [1] предположил, что число $s(n)$ свободных от сумм подмножеств натуральных чисел отрезка $[1, n]$ есть $O\left(2^{n / 2}\right)$. В [1] доказано, что число множеств, свободных от сумм, в отрезке $[n / 3, n]$ есть $O\left(2^{n / 2}\right)$. Н. Калкин в [2] доказал, что $s(n)=2^{n(1 / 2+o(1))}$. Для произвольного $\varepsilon>0$ обозначим через $s_{\varepsilon}(n)$ число множеств, свободных от сумм, в отрезке $[(1 / 4+\varepsilon) n, n]$. Нами доказывается следующая теорема.

Теорема 1. Для всякого $\varepsilon>0$ существует постоянная $c=c(\varepsilon)$ такая, что

$$
s_{\varepsilon}(n) \leqslant c 2^{n / 2} \text {. }
$$

\section{2. Определения и вспомогательные утверждения}

Подмножество $A$ вершин графа $G$ называется независимым, если подграф, порожденный множеством $A$, не содержит ребер. Число всех независимых множеств графа $G$ обозначим через $I(G)$. Назовем гранищей вершины $v$ в графе $G=(V, E)$ множество

$$
\partial v=\{u:(u, v) \in E\} .
$$

Границу подмножества $A$ графа $G$, определим как множество

$$
\partial A=\left(\bigcup_{v \in A} \partial v\right) \backslash A .
$$


Для подмножеств $\boldsymbol{A}$ и $\boldsymbol{B}$ аддитивной группы $H$ положим

$$
A+B=\{a+b: a \in A, b \in B\} .
$$

Заметим, что всюду далее логарифмы берутся по основанию 2.

Теорема 2 (Коши-Давенпорт [3]). Пусть $A, B$ - непустые подмножества элементов группы $H$ простого порядка. Тогда $A+B=H$ или $|A+B| \geqslant|A|+|B|-1$.

Лемма 1. Пусть в п-вершинном графе $G=(V, E)$ максимальная степень вершины не превосходит $k$, а доля вершин, степени меньшей $k$, не превосходит $\delta$. Тогда

$$
I(G) \leqslant 2^{(n / 2)(1+\delta+O(\sqrt{(\log k) / k}))} .
$$

Доказательство. С небольшими изменениями доказательство повторяет доказательство теоремы 2 из [4]. Для произвольного независимого множества $A$ построим множество $T$ с помощью следующей пошаговой процедуры.

Шаг 1. Пусть $u_{1}$ - произвольная вершина из $A$. Положим $T_{1}=\left\{u_{1}\right\}$.

Пусть сделано $m$ шагов и построено множество

$$
T_{m}=\left\{u_{1}, \ldots, u_{m}\right\}
$$

Шаг $m+1$. Если существует вершина $u_{m+1} \in A$ такая, что $\left|\partial u_{m+1} \backslash \partial T_{m}\right| \geqslant \varphi$, то полагаем

$$
T_{m+1}=T_{m} \cup\left\{u_{m+1}\right\} .
$$

В противном случае процесс заканчивается, и полагаем $T=T_{m}$.

Это множество $T$ будем называть $\varphi$-локализатором множества $A$. Число независимых множеств $A$, для которых множество $T$ является $\varphi$-локализатором, обозначим $I(G, T)$. Определим для каждого такого $T$

$$
D=D(T)=\{v \in V \backslash \partial T:|\partial v \backslash \partial T|<\varphi\} .
$$

Обозначим $D_{1}$ ту часть $D$, которая содержит вершины степени $k$. Рассмотрим двудольный подграф графа $G$ с долями вершин $D_{1}$ и $\partial T$. Степень каждой вершины из $D_{1}$ не меньше $k-\varphi$, а степень каждой вершины из $\partial T$ не меньше $k$. Поэтому

$$
\left|D_{1}\right|(k-\varphi) \leqslant|\partial T| k \text {. }
$$

Учитывая, что $|D|-\delta n \leqslant\left|D_{1}\right|$ и $|\partial T| \leqslant n-|D|$, получаем неравенство

$$
|D| \leqslant n(1+\delta) \frac{k}{2 k-\varphi} \text {. }
$$

Положим $\varphi=\sqrt{k \log k}$. Заметим, что для любого $A$ по построению

$$
|T| \leqslant|\partial A| / \varphi \leqslant n / \varphi .
$$

Следовательно, существует семейство $\Psi$ множеств $T \subseteq V$ такое, что для каждого независимого множества $A$ в нем найдется $\varphi$-локализатор множества $A$ и при этом

$$
|\Psi| \leqslant \sum_{i \leqslant n / \varphi}\left(\begin{array}{l}
n \\
i
\end{array}\right) \leqslant 2^{n \sqrt{(\log k) / k}} .
$$


В силу (3) и включения $A \subseteq D$

$$
I(G, T) \leqslant 2^{|D|} \leqslant 2^{(n / 2)(1+\delta+O(\sqrt{(\log k) / k}))} .
$$

Отсюда с учетом (4) получаем, что

$$
I(G) \leqslant \sum_{T \in \Psi} I(G, T) \leqslant 2^{(n / 2)(1+\delta+O(\sqrt{(\log k) / k})} .
$$

\section{3. Доказательство теоремы 1}

Поскольку $s_{\varepsilon}(n+1) \leqslant 2 s_{\varepsilon}(n)$, утверждение (1) равносильно аналогичному утверждению для четных $n$. Нам удобно будет считать, что $n$ четно. Обозначим через $S$ множество всех свободных от сумм подмножеств отрезка $[(1 / 4+\varepsilon) n, n / 2]$. Заметим, что каждое подмножество $C$ отрезка $[n / 2+1, n]$ свободно от сумм, а число таких подмножеств равно $2^{n / 2}$. Поэтому достаточно оценить число тех $C \in S$, наименьший элемент которых не превосходит $n / 2$. Назовем $t$ глубиной множества $C$, если наименьший элемент $C$ равен $n / 2-t$. Подсемейство всех $C \in S$ глубины $t$ обозначим через $S_{t}$. Если $t_{0}-$ некоторое фиксированное число, то, очевидно,

$$
\sum_{t=0}^{t_{0}}\left|S_{t}\right| \leqslant c\left(t_{0}\right) 2^{n / 2}
$$

где $c\left(t_{0}\right) \leqslant 2^{t_{0}+1}$. Поэтому в дальнейшем, оценивая $\left|S_{t}\right|$, мы ограничиваемся значениями $t$, превышающими $t_{0}$, где $t_{0}-$ некоторое достаточно большое число. Положим

$$
Z_{t}=[n / 2-t, n / 2]
$$

Если $A \subset[1, n / 2]$, то пусть

$$
\mathscr{B}(A)=\{B \subseteq[n / 2+1, n]: A \cup B \in S\} .
$$

Тогда

$$
\left|S_{t}\right|=\sum_{A \subseteq Z_{t}}|\mathscr{B}(A)|
$$

Пусть

$$
\begin{aligned}
& S_{t}^{\prime}=\left\{C \in S_{t}:\left|C \cap Z_{t}\right| \leqslant \log t\right\}, \\
& S^{\prime}=\bigcup_{t_{0}<t \leqslant(1 / 4-\varepsilon) n} S_{t}^{\prime} .
\end{aligned}
$$

Для оценки $\left|S_{t}^{\prime}\right|$ заметим, что для каждого $A \subseteq Z_{t}$ и любого $j=1, \ldots, t$ из каждой пары чисел $(n / 2+j, n+j-t)$ множеству $B \in \mathscr{B}(A)$ принадлежит не более одного числа. Отсюда следует, что

$$
|\mathscr{B}(A)| \leqslant\left(\frac{3}{4}\right)^{t} 2^{n / 2}
$$


Поскольку для достаточно большого $t$

$$
\left|\left\{A \subseteq Z_{t}:|A| \leqslant \log t\right\}\right| \leqslant 2^{\log ^{2} t},
$$

в силу (5) и (6)

$$
\begin{aligned}
\left|S^{\prime}\right| & =\sum_{t=t_{0}+1}^{(1 / 4-\varepsilon) n}\left|S_{t}^{\prime}\right| \leqslant \sum_{t=t_{0}}^{\infty} \sum_{A \subseteq Z_{t},|A| \leqslant \log t}|\mathscr{B}(A)| \\
& \leqslant \sum_{t=t_{0}}^{\infty} 2^{\log ^{2} t}\left(\frac{3}{4}\right)^{t} 2^{n / 2} \leqslant 2^{n / 2-c_{1} t_{0}},
\end{aligned}
$$

где $c_{1}$ - абсолютная положительная постоянная.

Положим

$$
S_{t}^{\prime \prime}=S_{t} \backslash S_{t}^{\prime}, \quad S^{\prime \prime}=\bigcup_{t_{0}<t \leqslant(1 / 4-\varepsilon) n} S_{t}^{\prime \prime}
$$

Оценим $\left|S_{t}^{\prime \prime}\right|$. Пусть

$$
C \in S_{t}^{\prime \prime}, \quad A=C \cap Z_{t},
$$

а $m$ - минимальное число такое, что

$$
|A \cap[n / 2-t, n / 2-t+m]|=\lceil\log t\rceil .
$$

Через $\Gamma_{t}(A)$ обозначим граф с множеством вершин

$$
V=[n / 2+1, n / 2+t] \cup[n-t+1, n],
$$

в котором вершины $u$ и $v$ соединены ребром, если

$$
u-v \in A \cap[n / 2-t, n / 2-t+m] \text {. }
$$

Заметим, что для каждого $A \in Z_{t}$ и $B \in \mathscr{B}(A)$ множество $B \cap V$ является независимым в $\Gamma_{t}(A)$. Кроме того, $|V|=2 t$, число вершин степени $\lceil\log t\rceil$ равно $2 t-2 m$, а остальные $2 m$ вершин имеют меньшую степень. В силу (2)

$$
I\left(\Gamma_{t}(A)\right) \leqslant 2^{t+m+O(t \sqrt{\log \log t / \log t})} .
$$

Пусть $C \in S^{\prime \prime}, A=C \cap Z_{t}$, а $u$ и $v$ таковы, что $u+v \in[n / 2+1, n] \backslash V$, тогда $u+v \notin C \backslash V$. Положим

$$
\xi=\frac{4 \varepsilon}{1-4 \varepsilon}, \quad A_{\xi}=C \cap[n / 2-t / 2-\xi t, n / 2-t / 2], \quad k=\left|A_{\xi}\right| .
$$

Заметим, что $u+v \notin V$ для любых $u, v \in A_{\xi}$. Пусть $p$ - простое число, $n \leqslant p-1$, и $H_{p}$ - группа вычетов по модулю $p$. Ясно, что $A_{\xi} \subset H_{p}$ и сумма любых двух элементов из $A_{\xi}$ не превосходит $n$. По теореме 2 число различных чисел вида $u+v$, где $u, v \in A_{\xi}$ не меньше $2 k-1$. Пусть $Q(m, k)-$ семейство множеств $A \subseteq Z_{t}$ таких, что

$$
|A \cap[n / 2-t, n / 2-t+m]|=\lceil\log t\rceil, \quad|A \cap[n / 2-t / 2-\xi t, n / 2-t / 2]|=k .
$$


Тогда

$$
|Q(m, k)| \leqslant 2^{\log ^{2} t+t-(m+1)-\xi t}\left(\begin{array}{c}
\xi t \\
k
\end{array}\right) .
$$

Для каждого $A \in Q(m, k)$ справедливы неравенства

$$
|\mathscr{B}(A)| \leqslant 2^{n / 2-(2 k-1)-2 t} I\left(\Gamma_{t}(A)\right) \leqslant 2^{n / 2-t+m-2 k+O(t \sqrt{\log \log t / \log t})} .
$$

Из (8) и (9) следует, что

$$
\begin{aligned}
\left|S^{\prime \prime}\right| & =\sum_{t=t_{0}+1}^{(1 / 4-\varepsilon) n}\left|S_{t}^{\prime \prime}\right| \leqslant \sum_{t=t_{0}}^{\infty} \sum_{m=\lceil\log t\rceil}^{t} \sum_{k=0}^{\xi t} \sum_{A \in Q(m, k)}|\Re(A)| \\
& \leqslant 2^{n / 2} \sum_{t=t_{0}}^{\infty} \sum_{m=\lceil\log t\rceil}^{t} \sum_{k=0}^{\xi t}\left(\begin{array}{c}
\xi t \\
k
\end{array}\right) 2^{-2 k} 2^{-\xi t+\log ^{2} t+O(t \sqrt{\log \log t / \log t})} \\
& \leqslant 2^{n / 2} \sum_{t=t_{0}}^{\infty} 2^{\xi(\log 25-3) t+\log t+\log ^{2} t+O(t \sqrt{\log \log t / \log t})} \leqslant 2^{n / 2-c_{2} t_{0}}
\end{aligned}
$$

где $c_{2}$ - положительная постоянная, зависящая от $\varepsilon$. Теперь из (7) и (10) получаем, что

$$
s_{\varepsilon}(n)=|S| \leqslant 2^{n / 2}\left(1+c\left(t_{0}\right)+2^{-c_{1} t_{0}}+2^{-c_{2} t_{0}}\right)=c 2^{n / 2},
$$

где $c$ - постоянная, зависящая от $\varepsilon$.

\section{Список литературы}

1. Cameron P., Erdős P., On the number of integers with various properties. In: Number theory, Proc. 1st Conf. Can. Number Theory Assoc., Banff/Alberta (Mollin, R. A., Ed.) 1988. De Gruyter, Berlin, 1990, pp. 61-79.

2. Calkin N. J., On the number of sum-free sets. Bull. London Math. Soc. (1990) 22, 141-144.

3. Mann H. B., Addition theorems: the addition theorems of group theory and number theory. Interscience, New York, 1965.

4. Сапоженко А. А., О числе независимых множеств в расширителях. Дискретная математика (2001) 13, №1, 56-62.

Статья поступила 10.04.2002. 